\title{
Loi fédérale sur la transplantation: occasion ratée de solidarité
}

\author{
J. Martin*
}

\footnotetext{
* Le Dr Martin, ancien médecin cantonal vaudois, est membre de la Commission nationale d'éthique pour la médecine humaine. Il s'exprime ici à titre personnel.
}

Correspondance: PD Dr Jean Martin La Ruelle 6 CH-1026 Echandens
Début juin, le Conseil des Etats a adopté, après le Conseil national, une loi sur la transplantation, première du genre au niveau fédéral (quelques divergences restent à aplanir). Ce texte fixe de manière détaillée les procédures qui régiront en Suisse la transplantation d'organes, de tissus et de cellules, de manière à préserver les droits de chacun et à assurer une disponibilité uniforme au niveau du pays. Le besoin d'une telle législation est certain, compte tenu de la pratique de plus en plus fréquente des transplantations, avec le risque de dérapages notamment au plan de l'équité et de l'éthique. En général, le texte adopté correspond aux doctrines actuelles en Europe.

Cependant, il faut regretter qu'une occasion de promouvoir une meilleure solidarité en matière de santé publique ait été ratée; ceci dans la mesure où le législateur prévoit l'exigence du consentement explicite du donneur plutôt que le consentement présumé. Ici, qu'il soit clair qu'en ce qui concerne des donneurs vivants (auxquels on fait appel de plus en plus, notamment en vue de greffe de rein), il n'est pas question de se passer du consentement explicite et éclairé. De plus, s'agissant de donneurs potentiels qui seraient mineurs, il importe soit de prohiber le don, soit de le soumettre à des garanties et vérifications très précises. En rapport avec les modalités de consentement, je parle donc ci-dessous de la situation de patients en état de mort clinique.

Plusieurs cantons, dont Genève et Vaud, connaissent actuellement dans leurs législations (qui seront invalidées par la promulgation de la loi fédérale) la notion de consentement présumé. L'idée étant que, à une époque où on entend souvent parler de transplantation, et où la notion de directives anticipées du patient est de mieux en mieux connue, la personne qui ne veut pas que, le cas échéant, ses organes soient prélevés en vue de transplantation a tout loisir de le faire savoir autour d'elle: à ses proches, à ses médecins, par écrit ou par une disposition orale expresse. Si la personne n'a pas manifesté une opinion ferme à ce sujet, on présume alors qu'elle ne s'oppose pas à un tel prélèvement, si ce don peut sauver l'un ou l'autre de ses contemporains.

Dans nos pays, chacun bénéficie d'une longue liste de prestations assurées par la collectivité (par l'intermédiaire des pouvoirs publics), notamment dans les domaines médical et social. J'estime que cette loi apportait une occasion de promouvoir la solidarité. Cette occasion a été ratée. Qu'on nous comprenne bien: il ne s'agit pas d'imaginer que l'on puisse prélever d'office les organes d'une personne (en état de mort clinique) qui ne le voulait pas, ou dont les proches peuvent témoigner qu'elle ne le voulait pas. Par contre, si elle ne s'est pas exprimée, ne peut-on pas considérer aujourd'hui, dans l'optique de concrétiser une citoyenneté solidaire, qu'elle admet d'assumer une certaine responsabilité visà-vis de la collectivité. Dans le sens d'un ultime «service civique» sachant que, ce faisant, on évite des morts d'hommes, on donne à notre prochain gravement malade une solide chance de vivre des années encore dans de bonnes conditions. C'est dire que, après une carrière consacrée à la santé publique, je trouvais souhaitable, adéquat, logique, équilibré (considérant les intérêts des parties concernées et de la société) qu'on prévoie au niveau fédéral la notion de consentement présumé que connaissent jusqu'ici plusieurs cantons. En bref, il s'agissait d'admettre que «qui ne dit mot consent», étant entendu une fois encore (et cela module ce que l'application de ce principe pourrait avoir de rude) que chacun devrait aujourd'hui être informé de l'éventualité de prélèvements d'organes et de la possibilité de manifester que, pour soi-même, on s'y oppose.

Cette question doit être vue dans un contexte large. Par exemple en rappelant que, avec le Luxembourg, la Suisse est le pays où on est le moins enclin à accepter de donner ses organes. N'y a-t-il pas là, dans un domaine particulier une manifestation encore de cet individualisme exacerbé, de ce repli sur son quant à soi, qui hypothèque aujourd'hui, au plan général, l'avenir de notre pays? Que je précise encore que je connais bien les exquises sensibilités actuelles aux droits individuels et que j'en respecte évidemment le principe. Toutefois, je nous souhaiterais une société où l'on se souvienne un peu mieux que chacun a aussi des devoirs vis-à-vis de ceux qui l'entourent, au près et un peu plus loin. Se souvenir aussi, même si la formule est quelque peu emphatique, de John F. Kennedy lors de son investiture: «Ne demandez pas ce que votre pays peut faire pour vous, demandez ce que vous pouvez faire pour votre pays». 